\title{
An improved energy Minimum Connected Dominating Sets Construction Algorithm in MANET
}

\author{
Zifen YANG ${ }^{1, a}$, Guoqiang LI ${ }^{1, b}$ Li LIU $^{1, c}$ \\ ${ }^{1}$ School of Informatics, Linyi University, Linyi, 276005, China

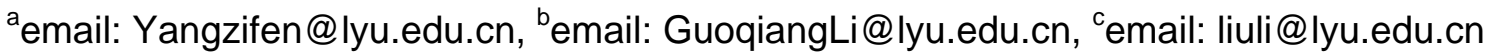

Keywords: Energy; Connected Dominating Sets; MANET

\begin{abstract}
In recent years, MANET gain a significant attention for the potential application in battlefield, disaster relief, traffic monitoring and environment monitoring. Construction and maintain of virtual backbone, and energy effective routing in mobile ad hoc wireless network come to be the basic and main problem in MANET. This paper proposed the efficient ENMCDS algorithm for constructing the minimum connected dominating set, the algorithm in most cases can quickly generate a near optimal size of ENMCDS. Theoretical analysis and simulation results show the superiority of the algorithm over other algorithms in terms of the CDS size and energy consumption.
\end{abstract}

\section{Introduction}

As a consequence of such as earthquake or flooding, many of the infrastructures have been damaged. Therefore, it is hard to communicate solution message with each other through infra-structured networks [1]. Adhoc are the collection of wireless mobile hosts (also called nodes) such as notebook, mobile phone or other portable equipment can communicate with each other by wireless channels [2]. MANET can provide an instant and distributed peer to peer ad hoc communication between equipment. Hence, MANET is more and more utilized for disaster relief operations. However, two nodes of a MANET can communicate directly only and only if they are within radio transmission ranges of each other. So, multi-hop routing is the only choice in a MANET. MANET routing protocols can be divided into flat routing and hierarchical routing protocol according to the logic of the network structure. In the flat routing protocol, all the nodes of MANET have equal status. A routing protocol for MANET uses a broadcasting scheme to discover a rout. Hence, node in network will repeat the sending or receiving broadcast signal [3]. The flooding of broadcast signal leads to congestion and also consumes more battery power. Also, as the topology is dynamic, touting tables need to be update frequently. Hence, we design a hop communication protocols the stability of path is an important criterion must to be considered.

A possible method to reduce the energy consumption in ad hoc network is hierarchical routing that is backbone-based routing. This also is to investigate in this paper. When the sender of the message want to send some message to destination, it send message to the backbone. Any node on the backbone examines the destination in the message, and if this destination is not one of its neighbors, it floods the message to other backbone nodes other than the sender. Therefore, the number of update messages for routing is also significantly reduced. A clear requirement is that the backbone nodes should be connected and that every node should be in the backbone or a neighbor to a backbone node. The connected backbone we called it as connected dominate set(CDS).Routing based on CDS have lot of advantage such as restrict search space. Thus, flooding storm can be avoided also significant route overhead can be reduced. And less energy can be consumed. In this paper we proposed a novel method to construct the backbone of MANET, which is CDS.

Section 2: the related work of routing protocol about energy, mobility and soon.

Section 3: Explains the system model and definition

Section 4: Explains our algorithm and route discovery using SON-MCDS

Section 5: The simulation results of the proposed work are presented

Section 6: the conclusion of our work. 


\section{Related works}

In this section, we provide the background on the overview constructing MCDS.

\subsection{Network modeling and related definitions}

Usually, a graph is used to represent the network model of wireless network .In this paper we utilize unit disk graph (UDG) as model of a MANET.

Definition 1 (Unit Disk Graph (UDG)) G (V, E) is UDG if $\forall u, v \in V$ there is a bidirectional edge between $\mathrm{u}$ and $\mathrm{v}$ if and only of $\operatorname{Eudist}(u, v) \leq 1$.

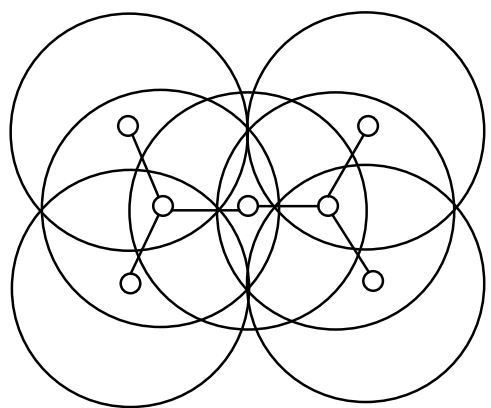

Fig1 unit disk graph model

Definition2: (Dominating Set ( $D S$ )) Given graph $G=(V, E), V^{\prime} \in V$ is a $D S$ of $G=(V, E)$, only if $\forall(u, v) \in E$, either $u \in V^{\prime}$ or $v \in V^{\prime}$ is true.

Definition3: (Connected Dominating Set (CDS ) $(C \in V)$ is a CDS of $G$ if (1) $C$ is a $D S$ and (2) a graph induced by $C$ is connected.

\subsection{Related works on finding MCDS}

Due to the overhead of backbone node is larger, many methods to reduce the size of CDS .That can greatly interference and control the amount of information transmission [4]. In graph theory, finding the minimum CDS is NP-hard [5]. Many works has been done to construct Minimum CDS such as this literature. There are two kinds of method to construct CDS. One method is based on the reduction [6, 7], and the other one is based on the increase [8,9]. The first method construct a CDS from one node with maximum degree, and color it, then all of the neighbors of this node is colored with another color to formation initial CDS, then reduce some nodes by some rules to reduce the size of CDS .And another method first computes a dominate set and then selection some nodes to connect the nodes of dominate set.

The literature shows that the size of CDS which is constructed based on increasing is smaller than method with reduction. However, the tree structure based on CDS protocol is usually through the timer spanning tree, when the network size is larger, the tree based CDS protocol requires more time in the construction of CDS [10]. And construction and maintenance of virtual backbone in wireless network is very important [11].

Therefore, we are looking for a protocol to construct CDS fast and we know the advantages of tree based on CDS protocol. This chapter uses a CDS protocol based on network increase. A control for the first set of DS network, and then through the tree based approach to connect all the nodes in the DS.

\section{Formation EN-MCDS}

In this section, we propose a three-phase algorithm to construct and repair N-MCDS.

This chapter uses a CDS protocol based on network increase. A control for the first set of DS network, and then through the tree based approach to connect all the nodes in the DS. In the first phase, finding DS by analysis the relation between neighbor set .Then a Steiner tree is constructed to connecting all of nodes in Steiner tree. Thus CDS is formed. At last, the size of CDS is getting smaller based on the reduction. Thus, our algorithm consists of three stages, constructing DS, constructing Steiner tree and reducing. 


\section{1 notations and definitions}

Some notations and definitions which are used in our algorithm is show as follow:

$N[u]$ : The neighbor set of node $u$, that is $N[u]=N(u) \bigcup\{u\}$.

List : A queue with non-increasing order. All the nodes of $N[u]$ are stored in List with the frequency of occurrence, that is the first element in $N[u]$ is the node with the most of frequency occurrence. The main purpose of dequeue operation of List is to get the first element of List .

Steiner tree $:=(V, E$, cost $)$ be a graph with a nonnegative cost function on its edges. Any tree in $G$ spanning a given set of terminals $s \in V$ is called a Steiner tree. And the cost of a tree is defined to be the sum of its edge costs. The Steiner Tree Problem (STP) seeks a minimum-cost Steiner tree. Note that a Steiner tree may contain non-terminal vertices and these are referred to as Steiner points.

$D$ : Dominate set of network which is constructing in first stage. Initially, the set of $D$ is null.

\section{2 formation EN-MCDS}

In this section we will formation EN-MCDS in three stages.

\section{A. constructing DS}

Step1: every node transmits a hello message to its neighbors. And $N[u]$ is consists of the one hop neighbor of node $u$.

Step2: every node get $N[u]$, traverse the node's neighbor set. All the nodes of $N[u]$ are stored in List with the frequency of occurrence.

Step3: Perform dequeue operation to List. Let $u$ is the node which we get from dequeuer operation. $u$ is colored with black. And all the nodes in $N[u]$ but $u$ is colored with Gray. And $D \leftarrow\{v\}$.

Step4: Perform dequeue operation again. Let $v$ is the node which we get from dequeuer operation. $v$ is colored with black. And all the nodes in $N[v]$ but $v$ is colored with Gray. And $D \leftarrow\{v\}$.

Step5: Iterative step 4 until there is no node with color white.

Step6: $D$ consists of all the black nodes.

\section{B. constructing Steiner tree}

Step1: choose the largest $N[u]$ as the leader of tree.

Step2: if there is no path between $u$ and leader then create the shortest path via GRAY node.

Step3: if there is a path between $u$ and leader Iterative step 2.

CDS consist of all the black nodes.

C. reducing CDS

Step1: Check whether the black leaf node $i$ is only controlled by gray node in the Steiner tree.

Step2. If there is no such grey node, delete $i$ from $D$.

Step3. If no such grey node our method go back to perform Step1.

Step4.This perform executing until check all of the nodes in network. MCDS consists of all the black nodes.

\section{Analysis and simulation}

\subsection{Analysis}

Theorem1: we can get a CDS from our algorithm.

Proof. NMCDS algorithm is divided into three stages; DS is obtained from the first stage operation. All nodes in the network are dominated by the node in DS. Then a Steiner tree is constructed according to the characteristics of Steiner tree, all nodes that are connected through the 
Steiner tree in DS network that is CDS. The size of CDS is reduced in the third stage .And we also guarantee that each node has at least one gray black neighbor nodes .so this step is not damage characteristics. At the end of the algorithm, all nodes in the network are dominated by a small size of CDS. The NMCDS algorithm is correct.

\subsection{Simulation}

In this section, we simulate the algorithm with Matlab program to prove the efficiency. The experiments are repeated for 50 trials with different network sizes and speeds of mobile nodes. The experimental configuration is listed as follows: a rectangular grid of ranging from $5 * 5$ to $1000 * 1000$ and 5-200 points are distributed randomly over the grid. The other parameters are listed as follows:

Parameter

value

Area size

$1000 * 1000$

Traffic type

Constant Bit Rate (CBR)

Packet size

512 bytes

MAC protocol

IEEE 802.11

Transmission range

$250 \mathrm{~m}$

Bandwidth

2Mbps

Queue size

50 packets

Experiments are repeated for 30 trials with different network sizes, load conditions and mobility. We compared the simulation results to the AEBM-CDS [12] with our scheme.

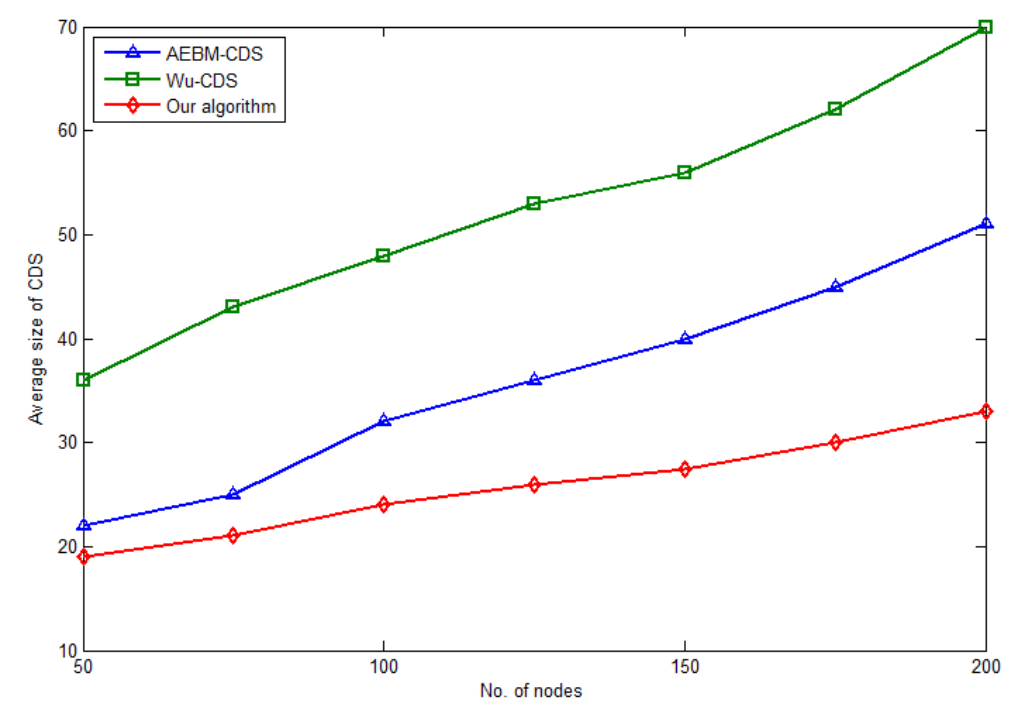

Fig2 average of CDS size

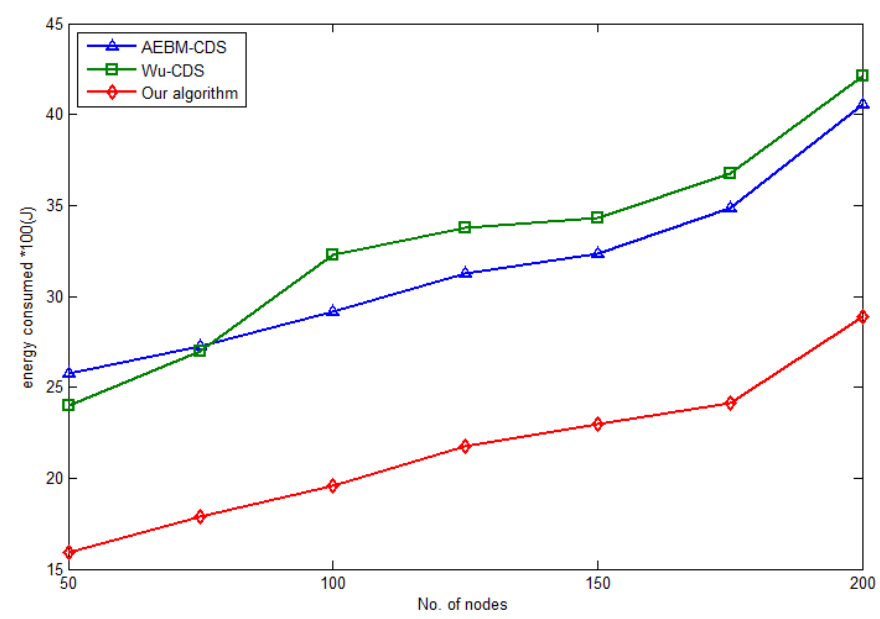

Fig3 energy consumption 
We note that, the algorithm of our method gets a good result of minimum size CDS as showed fig2 and fig3; it can guarantee an optimal network performance. The total energy consumption of the protocols increases with the increasing node speed.

\section{Conclusion}

This paper is aiming at the problem of lacking infrastructure in MANET, this paper proposed an energy balanced minimum connected dominating sets ( MCDS) construction algorithm. And we confirm the energy efficiency of the DET-CDS and the size of CDS is superior to others.

\section{Acknowledgement}

In this paper, the research was sponsored by the Nature Science Foundation of Shandong Province (Project No. ZR2014FL013). The research also was sponsored by National Training Programs of Innovation and Entrepreneurship for Undergraduates (project No.201410452039). The research also was sponsored by the Excellent Course Project of Colleges and Universities in ShanDong Province (Project No. 2013BK109).

\section{References}

[1] Jang, H., Lien, Y., \& Tsai, T. Rescue information system for earthquake disasters based on MANET emergency communication platform. In IWCMC 09. 2009 623-627.

[2] A. Ephremides, J. Wieselthier, D. Baker. A design concept for reliable mobile radio networks with frequency hopping signaling [C]. In Proc of the IEEE, 1987, 75(1): 56-73.

[3]Chang,Yu-Liang, and Ching-Chi Hsu. "Routing in wireless/mobile ad-hoc networks via dynamic group construction." Mobile Networks and Applications 2000: 27-37.

[4] Wu, Jie, and Li Hai-lan. "On calculating connected dominating set for efficient routing in ad hoc wireless networks." Proceedings of the 3rd international workshop on Discrete algorithms and methods for mobile computing and communications. ACM, 1999.

[5] Michael, R. Garey, and S. Johnson David. "Computers and intractability: a guide to the theory of NP-completeness." WH Freeman \& Co., San Francisco 1979.

[6] Wan, Peng-Jun, Khaled M. Alzoubi, and Ophir Frieder. "Distributed construction of connected dominating set in wireless ad hoc networks."INFOCOM 2002. Twenty-First annual joint conference of the IEEE computer and communications societies. Proceedings. IEEE. Vol. 3. IEEE, 2002.

[7] Li yong, Ying-shu, "On greedy construction of connected dominating sets in wireless networks." Wireless Communications and Mobile Computing 5.8 2005: 927-932.

[8] Shalom, Menny "Energy level alignment in CdS quantum dot sensitized solar cells using molecular dipoles." Journal of the American Chemical Society 2009: 9876-9877.

[9] Dai, Fei, and Wu Jie. "An extended localized algorithm for connected dominating set formation in ad hoc wireless networks." Parallel and Distributed Systems. IEEE Transactions on15.10 2004: 908-920.

[10] Zhou, Dong, Min-Te Sun, and Ten-Hwang Lai. "A timer-based protocol for connected dominating set construction in ieee 802.11 multihop mobile ad hoc networks."Applications and the Internet, 2005. Proceedings. The 2005 Symposium on. IEEE, 2005.

[11] Shukla, K. K., and S. Sah. "Construction and maintenance of virtual backbone in wireless networks." Wireless networks 19.5 2013: 969-984. 
[12] Lu Deng-yue; FAN Jian-xi; LIU Wen-jun; An Energy Balanced Minimum Connected Dominating Sets Construction Algorithm Journal of Chinese Computer Systems Vol35 No3 2014. 383-387 